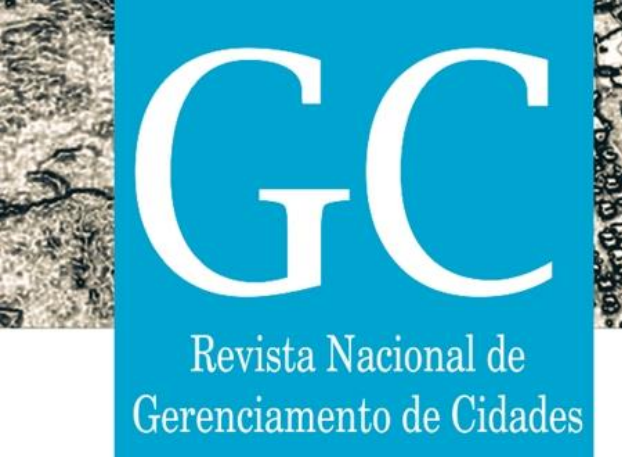

ISSN 2318-8472

v. 07 , n. 51, 2019

\title{
Zoneamento de áreas de risco: uma visão para a Agenda 2030
}

Risk areas zone: a vision for Agenda 2030

Zona de zonas de riesgo: una visión para la Agenda 2030

Francisca Ranielly de Brito Macêdo Mestranda em Geografia, UERN, Mossoró/RN, Brasil,

f.raniellydbm@gmail.com

Alfredo Marcelo Grigio

Professor Doutor, UERN, Mossoró/RN, Brasil alfredogrigio1970@gmail.com

Márcia Regina Farias da Silva Professora Doutora, UERN, Mossoró/RN, Brasil mreginafarias@hotmail.com 


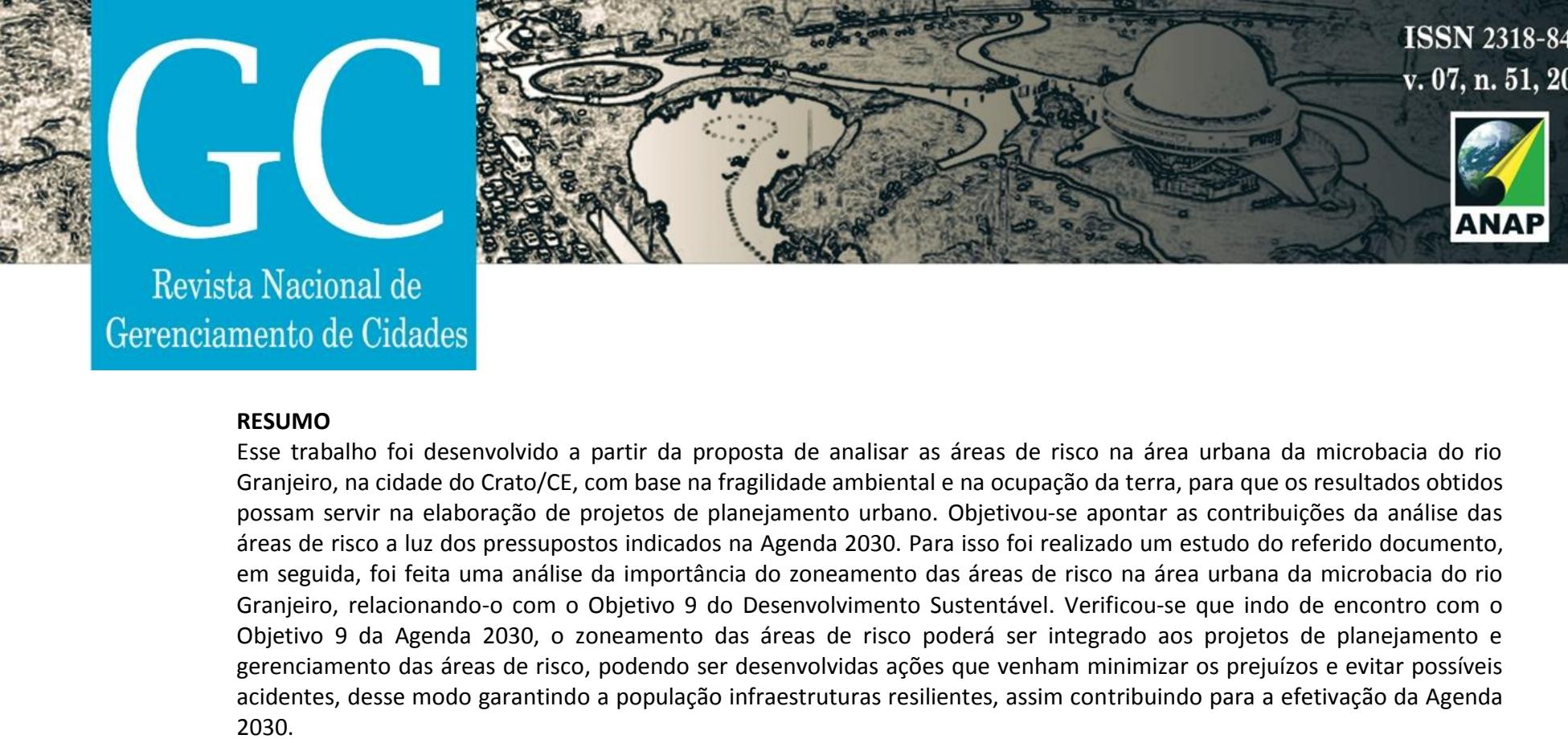

RESUMO

Granjeiro, na cidade do Crato/CE, com base na fragilidade ambiental e na ocupação da terra, para que os resultados obtidos possam servir na elaboração de projetos de planejamento urbano. Objetivou-se apontar as contribuições da análise das 2030.

Palavras-chave: Fragilidade Ambiental. Infraestrutura. Planejamento Urbano.

\section{ABSTRACT}

This work was developed from the proposal to analyze the risk areas in the urban area of the Granjeiro river basin, Crato/ $C E$, based on environmental fragility and land occupation, so that the results can serve in the elaboration of projects of urban planning. The objective was to point out the contributions of the risk areas analysis in light of the assumptions indicated in Agenda 2030. For this purpose, a study of this document was carried out, followed by an analysis of the importance of risk area zoning in the urban area of Granjeiro watershed, relating it to Sustainable Development Goal 9. Meeting the 2030 Agenda Objective 9, risk area zoning can be integrated into risk area planning and management projects, and actions can be developed that minimize losses and prevent possible accidents. guaranteeing resilient infrastructures, thus contributing to the realization of the 2030 Agenda.

Keywords: Environmental fragility. Infrastructure. Planning Urban.

\section{RESUMEN}

Este trabajo se desarrolló a partir de la propuesta de analizar las áreas de riesgo en el área urbana de la cuenca del río Granjeiro, Crato / CE, con base en la fragilidad ambiental y la ocupación de la tierra, para que los resultados puedan servir en la elaboración de proyectos de planificación urbana El objetivo fue señalar las contribuciones del análisis de áreas de riesgo a la luz de los supuestos indicados en la Agenda 2030. Para este propósito, se llevó a cabo un estudio de este documento, seguido de un análisis de la importancia de la zonificación del área de riesgo en el área urbana de Cuenca hidrográfica del Granjeiro, relacionándola con el Objetivo de Desarrollo Sostenible 9. Al cumplir el Objetivo 9 de la Agenda 2030, la zonificación del área de riesgo se puede integrar en los proyectos de planificación y gestión del área de riesgo, y se pueden desarrollar acciones que minimicen las pérdidas y eviten posibles accidentes. Garantizando una infraestructura resistente a la población, contribuyendo así a la realización de la Agenda 2030.

Palabras clave: Fragilidad ambiental. Infraestructura. Planificacion Urbana. 


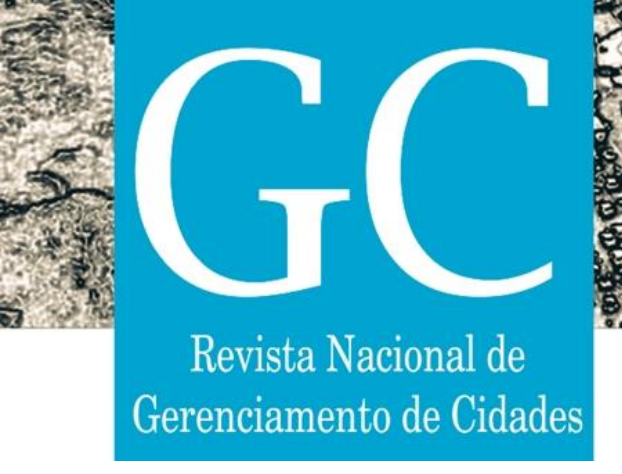

A ONU (2015) definiu 17 Objetivos de Desenvolvimento Sustentável (ODS) como parte da Agenda 2030 (Figura 02). Esses objetivos buscam concretizar os direitos humanos de todos e alcançar a igualdade de gênero, empoderamento das mulheres e meninas. Estes objetivos são integrados e indivisíveis os quais se equilibram em três dimensões do desenvolvimento sustentável: a economia, a social e a ambiental.

Figura 02 - Objetivos de Desenvolvimento Sustentável
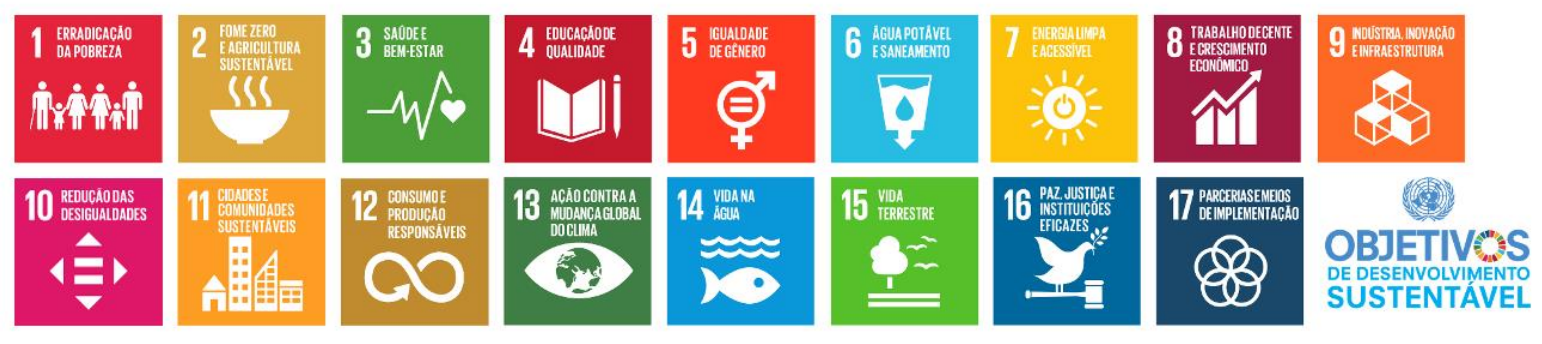

Fonte: ONU, 2015.

Os objetivos e as metas são resultados de mais de dois anos de consulta pública intensiva e envolvimento junto a sociedade civil, como também outras partes do mundo interessadas em contribuir, ressaltando ainda que foi dada uma atenção especial às dos mais pobres e mais vulneráveis, esta agenda é guiada pelos propósitos e princípios da Carta das Nações Unidas, incluindo o pleno respeito pelo direito internacional (ONU, 2015).

Dentre os 17 objetivos dos ODS, será aqui destacado o objetivo 9 que é o de Indústria, Inovação e Infraestrutura que tem como metas: construir infraestruturas resilientes, promover a industrialização inclusiva e sustentável e fomentar a inovação. Diante disso, a discussão desse trabalho dará ênfase a meta de construir infraestruturas resilientes.

O conceito de infraestrutura é abrangente, mas em termos gerais, pode-se considerar como infraestrutura o conjunto de atividades e estruturas que se baseiam no desenvolvimento de todas as atividades, portanto esse conceito pode variar de acordo com a área e o escopo ao qual se refere (ONU, 2015).

Devido a variação desse conceito, a ONU trata a infraestrutura em cinco vertentes, sendo elas: infraestrutura de qualidade, infraestrutura para o bem-estar humano, infraestrutura para o desenvolvimento econômico, infraestrutura resiliente e infraestrutura sustentável.

Como já foi mencionado anteriormente, neste estudo a discussão reside na questão da infraestrutura resiliente, sendo definida pela ONU (2015), como aquela capaz de resistir, absorver, acomodar ou se recuperar de impactos como desastres naturais de maneira rápida e eficiente, inclusive pela preservação e restauração de suas estruturas e funções básicas essenciais.

A infraestrutura resiliente representa o potencial do serviço urbano, rural, natural e de qualquer outra natureza em absorver e se recuperar do desastre sem gerar transtorno aos diversos grupos populacionais. A resiliência de uma comunidade depende dos seus recursos e de sua capacidade de se organizar antes, durante e após a ocorrência dos desastres naturais (ONU, 2015). O quadro a 


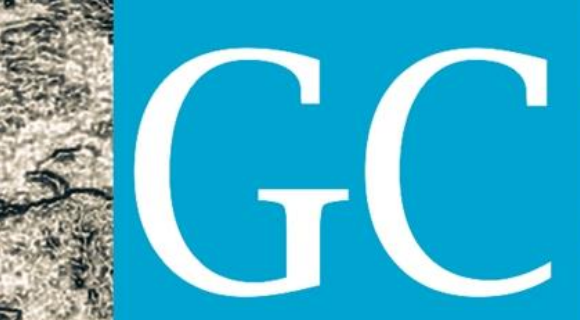

Revista Nacional de

Gerenciamento de Cidades

Esse trabalho contribui na ampliação das discussões acerca dos Objetivos de Desenvolvimento Sustentável, como também sobre a questão das ocupações em áreas de risco e os impactos das ações antrópicas nessas áreas. Com isso, esse debate poderá chegar a população mais vulnerável, levando-os a buscar mais informações e orientações de como agir de forma correta diante de uma situação de risco.

O estudo aqui proposto é fundamental para identificar as potencialidades e fragilidades do meio natural e urbano, desse modo, podem ser tomadas medidas capazes de eliminar ou reduzir os problemas dessas áreas. A análise da fragilidade do meio natural se faz fundamental para dar suporte no planejamento urbano, para traçar planos e implantar medidas para um melhor uso e ocupação da área, de modo que os impactos sejam os menores possíveis.

Portanto, o zoneamento das áreas de risco irá servir como ponto de partida para outras pesquisas relacionadas a questão ambiental que venham ser desenvolvidas na região e provocar novas discussões sobre a temática. O material produzido pode subsidiar os projetos de planejamento do município, facilitando o desenvolvimento de práticas que correspondam as necessidades de cada área, assim auxiliando alcançar o Objetivo 9 da Agenda 2030.

\section{REFERÊNCIAS}

BOTELHO, R. G. M. Bacias hidrográficas urbanas. In: GUERRA, A. J. T. (Org.). Geomorfologia urbana. Rio de Janeiro: Bertrand Brasil, 2011. Cap. 3.

BRASIL, Ministério das Cidades/Instituto de Pesquisas Tecnológicas - IPT. Mapeamento de Riscos em Encostas e Margens de Rios. Brasília: Ministério das Cidades/Instituto de Pesquisas Tecnológicas - IPT, 2007.

BRASIL. Objetivos de Desenvolvimento do Milênio - Relatório Nacional de Acompanhamento. Brasília: IPEA, 2004. Disponível em: <http://www.odmbrasil.gov.br/odmbrasil/arquivos/odmrelatorio-nacional-de-acompanhamento-2004> Acesso em: 19 de jul. de 2019.

BRITO, D. da S.; SILVA, J. M. O. Análise hidrográfica do micro bacia do Rio Granjeiro/Crato Ceará. Revista Geonorte. Edição Especial 2, v. 1, n. 5, p. 359-369, 2012.

CANO, W. Urbanização: sua crise e revisão de seu planejamento. Revista de Economia Política, Campinas, v.9, n.1, p. $62-82$, jan. 1989.

CARDOSO, E. J.; SANTOS, M. J. dos; CARNIELLO, M. F. O processo de urbanização brasileiro. In: ENCONTRO LATINO AMERICANO DE PÓS-GRADUAÇÃO - UNIVERSIDADE DO VALE DO PARAÍBA, 11., 2011, São José dos Campos. Anais... São José dos Campos, 2011.

CRUZ, L. M; JÚNIOR, J. F. P; RODRIGUES, S. C. Abordagem Cartográfica da Fragilidade Ambiental na Bacia Hidrográfica do Glória - MG. Revista Brasileira de Cartografia. n. 62/03, (ISSN 0560-4613), 2010.

CRUZ, M. A. S.; TUCCI, C. E. M. Avaliação dos cenários de Planejamento na Drenagem Urbana. Revista Brasileira de Recurso Hídricos. v.13, n.3, p. 59-71, 2008. 


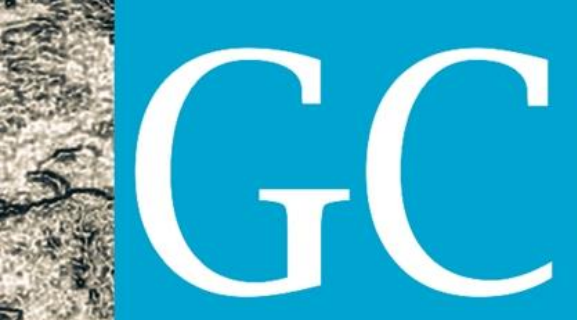

Revista Nacional de

Gerenciamento de Cidades

FREITAS, C. F. S. Ilegalidade e degradação em Fortaleza: os riscos do conflito entre a agenda urbana e ambiental brasileira. urbe. Revista Brasileira de Gestão Urbana (Brazilian Journal of Management). v.6, n.1, p. 109-125. jan./abr. 2014

GUERRA, A. J. T.; MARÇAL, M. dos S. Geomorfologia Ambiental. 7. ed. Rio de Janeiro: Bertrand Brasil, 2015, 190p.

IBGE - Instituto Brasileiro de Geografia e Estatística. IBGE Cidades: Crato. Disponível em: $<$ https://cidades.ibge.gov.br> Acesso em: 22/08/2019.

IPECE. Instituto de Pesquisa e Estratégia Econômica do Ceará. Perfil Básico Municipal de Crato 2017. Disponível em: < https://www.ipece.ce.gov.br> Acesso: 22/08/2019.

JORGE, M. do C. O. Geomorfologia urbana: conceitos, metodologias e teorias. In: GUERRA, A. J. T. (Org.). Geomorfologia urbana. Rio de Janeiro: Bertrand Brasil, 2011. Cap. 3.

JULIÃO, R. et al. Guia metodológico para a produção de cartografia municipal de risco e para a criação de Sistemas de Informação Geográfica (SIG) de base municipal. Autoridade Nacional de Proteção Civil, 2009.

MONTAÑO, M.; OLIVEIRA, I. S. D.; RANIERI, V. E. L.; SOUZA, M. P.; FONTES, A. T. O papel do instrumento zoneamento ambiental no processo de licenciamento de atividades: o caso do novo aterro sanitário do município de Piracicaba (SP). In: CONGRESSO BRASILEIRO DE CIÊNCIA E TECNOLOGIA EM RESÍDUOS E DESENVOLVIMENTO SUSTENTÁVEL, 2004, Florianópolis. Anais... Florianópolis: ICTR, 2004, p. 1523-1534.

NAÇÕES UNIDAS NO BRASIL - ONU BR. Plataforma Agenda 2030 - Acelerando as transformações para a Agenda 2030 no Brasil - Objetivo 9: Construir infraestruturas resilientes, promover a industrialização inclusiva e sustebtável e fomentar a inovação. Disponível em:

<https://nacoesunidas.org/pos2015/ods6/> Acesso em: 19 de jul. de 2019.

NUNES, L. H. Urbanização e desastres naturais: abrangência América do Sul. São Paulo. Oficina de Textos, 2015.

PARIZZI, M. G. Desastres naturais e induzidos e o risco urbano. Geonomos. Minas Gerais, v. 22, n. 1, p. 1 - 9. 2014.

RIBEIRO, S. C. Suscetibilidade aos processos erosivos superficiais com base na dinâmica geomorfológica na microbacia do Rio Granjeiro, Crato/CE. 2004. 148 p. Dissertação de Mestrado, Rio de Janeiro: UFRJ/PPGG.

ROBAINA, L. E. de S. Espaço urbano: relação com os acidentes e desastres naturais no Brasil. Ciência e Natura. Santa Maria, v. 30, n.2, p. 93 - 105. 2008.

ROSS, J. L. S. Análise Empírica da Fragilidade dos Ambientes Naturais e Antropizados. Revista do Departamento de Geografia, São Paulo, n. 8, p.63-74, 1994.

SANTOS JÚNIOR, V. J. dos.; SANTOS, C. O. A evolução da urbanização e os processos de produção de inundações urbanas. Estação Científica, Macapá, v.3, n.1, p. 19-30, jan./jun. 2013. 


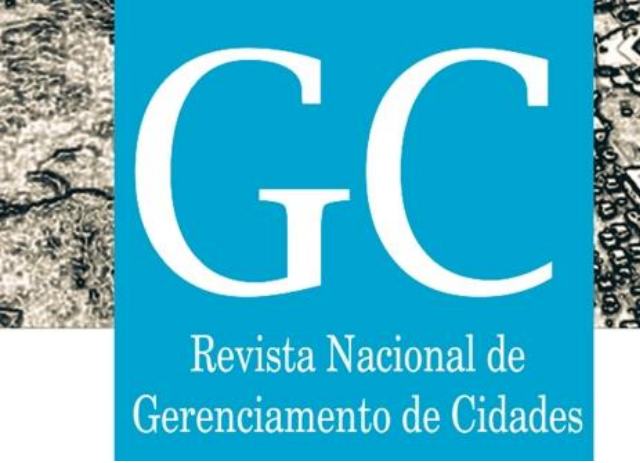

SOUZA, M. L. de. Mudar a cidade: uma introdução crítica ao planejamento e a gestão urbanos. Rio de Janeiro: Bertrand Brasil, 2008. 556p.

SPÖRL, C.; ROSS, J. L. S. Análise comparativa da fragilidade ambiental com aplicação de três modelos. GEOUSP - Espaço e Tempo, São Paulo, n.15, p.39-49, 2004.

TUCCI, C. E. M. Água no meio urbano. In: TUCCI, C. E. M. Água doce. Rio Grande do Sul: Instituto de Pesquisas Hidráulicas. 1997. Cap. 16.

VEYRET, Y. (org.). Os riscos: o homem como agressor e vítima do meio ambiente. São Paulo: Contexto, 2007.

ZAMPARONI, C. A. G. P. Risco e desastres naturais em ambiente urbano: o exemplo de Cuiabá/MT.

Revista Brasileira de Climatologia. v. 10, p. 7-20, 2012. 